\title{
Correction to "CXL146, a Novel 4H-Chromene Derivative, Targets GRP78 to Selectively Eliminate Multidrug-Resistant Cancer Cells"
}

In the above article [Bian T, Tagmount A, Vulpe C, Vijendra KC, and Xing C (2020) Mol Pharmacol 97:402-408; DOI: https://doi.org/10.1124/mol.119.118745], Amin Sobh was omitted from the byline. The correct byline for the article is Tengfei Bian, Abderrahmane Tagmount, Amin Sobh, Christopher Vulpe, Kavitha Chandagirikoppal Vijendra, and Chengguo Xing.

The authorship contributions of Dr. Sobh are as follows: Participated in research design, conducted experiments and performed data analysis.

The current affiliation of Dr. Sobh is Division of Hematology/Oncology, Department of Medicine, College of Medicine, University of Florida, Gainesville, Florida.

The HTML and PDF versions of the article have been corrected.

The authors apologize for any inconvenience this may have caused. 